Paper presented at the 1988 Synchrotron Radiation Instrumentation Meeting, Tsukuba, Japan, August 29 - Septembẹ.2, 1998

DE89 006182

\title{
MAGNETIC MEASUREMENTS ON INSERTION DEVICES AT NSLS ${ }^{+}$
}

Glenn Decker ", John Galayda ${ }^{*}$, Masashi Kitamura ${ }^{* *}$, Lorraine Solomon *

* National Synchrotron Light Source, Brookhaven National Laboratory, Upton, NY 11973 USA

** Hitachi Research Laboratory, Hitachi Ltd., 1-1, Saiwaicho 3 chome, Hitachi-shi, Ibaraki-ken, 317 Japan

Magnetic measurements on three insertion devices for use in the NSLS $x$-ray ring are described. Hall probes were used for fieid mapping, and rotating long coils were used to determine integrated multipole coefficients. Results are presented for an iron pole REC hybrid undulator, a vanadium permendur pole - REC hybrid wiggler and a 5 Tesla superconducting wiggler.

${ }^{+}$Work performed under the auspices of the U.S. Department of Energy.

\section{DISCLAIMER}

This report was prepared as an account of work sponsored by an agency of the United States Government. Neither the United States Government nor any agency thereof, nor any of their employees, makes any warranty, express or implied, or assumes any legal liability or responsibility for the accuracy, completeness, or usefulness of any information, apparatus, product, or process disclosed, or represents that its use would not infringe privately owred rights. Reference herein to any specific commercial product, process, or service by trade name, trademark, manufacturer, or otherwise does not necessarily constitute or imply its endorsement, recommendation, or favoring by the United States Government or any agency thereof. The views and opinions of authors expressed herein do not necessarily state or reflect those of the United States Government or any agency thereof.

\section{MASTER}

OISTAIBUTION OF THIS DOCUMEAT IS HMLLRTEO 


\section{II:TRODUCTION}

The phase II construation project at the National Synchrotron Light Source (NSLS) includes the design and construction of several insertion device magnets to be installed in the X-ray ring. Ultimately, five devices will be operational in the $x$-ray ring. Presently, both the mini-undulator at beam line $\mathrm{X} 13$, and the soft $\mathrm{X}$-ray undulator, or $\mathrm{SXU}$, at $\mathrm{X} 1$ are in the ring. The hybrid wiggler, or HBW, for beam line X25 is undergoing magnetic measurements. Final system testing is birg done on the superconducting wiggler, or SUW, for X17, and a HBW magnet for X21 is under construction. Shown in table 1 are fundamental parameters for SXU, HBW, and SUW.

At present, $x$-iay optics studies are being performed using the $S X U$ magnet, with the end goal being to perform photoemission microscopy and biological soft $\mathrm{x}$-ray imaging. The mini-undulator, a ten period prototype of the SXU magnet, has already been used as a radiation source (1). Crystallography, EXAFS and topography will be performed at $\mathrm{X} 17 \mathrm{~B} 1$. Medical research, mainly angiography, will be done at X17B2, and X17C will be used for high pressure research (2). The existing .BW nugnet will be used for high momentum transfer resolution $x$-ray scattering, and the HBW nuagnet under construction will be used for higl energy resolution $x$-ray scattering.

\section{MVEASURENITIT TESHNIOUE}

Fill probe measuremcits were straight forward once a precisely constructed probe carriage was available. The error in the probe position had to be small compared to the mignet period, and must not accumulate over the magnet length. The SXU carriage had 4 meters of motion, and its position was real back with an accuracy of \pm .001 inches using a transducer. The SUW carriage was simply constructed, with a placement accuracy of \pm .005 inches. The IIEW magnets will be measured using a 2.5 meter long air bearing 
table with a lead screw that locates the carriage with accuracy \pm .001 inches. The Hall probes used had an active area with .04 inches diameter.

The voltage induced in "long coil" windings when rotated yields integrated magneric multipole coefficients. The long coils used were rectangular, typically two centimeters wicle and several meters long, and supported on in G-10 tube. The long coil was piacedi along the $z$-axis of the magnet (figure 1), and is iong enough to extend beyond the magnet fringe fields. For a continuously rotating coil, the multipole coefficents $c_{n}$ are related to the induced voltage according to (3)

$$
V=-M \frac{d}{d t} \operatorname{Re}\left(\sum_{n=1}^{\infty} c_{n}\left[z_{2}^{n}-z_{1}^{n}\right]\right\}
$$

where $z_{k}=x_{k}+i y_{k}, k=1,2$ defines the transverse locations of the two halves of the long coil e.g. $z_{1}=-z_{2}=r \exp (i \omega)$, and $M$ is the number of turns. The symbol $z_{\dot{k}}$ used here is not to be confused with the $\mathrm{z}$ - axis of figure 1 .

The transverse components of the magnetic field, integrated along the $x$-axis are given by

$$
b_{x}-i b_{y .}=i \sum_{n=1}^{\infty} n c_{n}(x+i y)^{n-1}
$$

which effectively defines the multipole coefficients.

A continuously rotating coil was used to measure the SUW coefficients. For the SXU, a simpler method of flipping the coil by 180 degrees, and using a voltage integrator to measure the change in magnetic flux was used. The relation between the integrated voltage and the multipole coefficients is easily found by integrating equation (1). 
Problems with the long coil technique are related to construction imperfections. The measured values of the multipole components should in principle have been independent of the location of the long coil along the $\mathbf{z}$ - axis. Nonuniformities of the long coil windings along their length cause the measured values to oscillate as the long coil is moved along the $\mathrm{z}$ - axis, requiring systematic correction.

\section{THE SXU MAGNET}

The SXU magnet is constructed of blocks of rare earth cobalt (REC) permanent magnet material sandwiched between iron pole pieces, in a hybrid configuration ${ }^{(4)}$. Figure 1 shows schematically a Hall probe measurement arrangement for the SXU magnet.

Prior to assembly of the SXU magnet, the magnetization of the individual blocks of permanent magnet material was determined using Helmholtz coils in conjunction with a voltage integrator. Averages over eight different block orientations were performed to determine the major (z) and minor ( $y)$ components of the magnetization.

Instrumental in the block sorting procedure were "transfer function" measurements. Here, a single block was loaded into a magnet slot and Hall probe scans were made. For each of two orientations, i.e. the major magnetization component along either 2 or $\hat{y}$, a measurement was made for both the high and low positions in the slot. Boundary conditions were fixed by locating either an empty magnet beam with no permanent magnet material, or an iron plate to provide a mirror image, directly above the beam containing the single block. In all cases, the residual field after block removal was measurec and subtracted.

An important result of the transfer function measurements was the fact that the minor component field integral for a block in the low position is comparable to that for a 
block in the high position. Apparently, the portion of magnet material extending beyond the pole piece near the iron back plate has fields which are not well shielded by the pole material. This effect was also observed in the HBW magnet.

The transfer functions were essential to the sorting of magnet blocks, since they could be superposed and weighted according to the Helmholtz coil block data, resulting in a prediction for the field profile. The superposition hypothesis rests on the assumption that the iron poles form magnetostatic equipotentials. This is a reasonable assumption, because the peak pole tip fields in the SXU magnet are about $6.6 \mathrm{kG}$, where the permeability is still quite large for iron.

A second assumption for superposition is that the magnet is infinitely long, since the transfer function data were taken in the middle of the magnet. For an infinitely long magnet, the major component produces no net field integral because of symmetry. For this reason, it was decided to leave three slots unloaded at each end of the magnet, so that the magnet ends are in an essentially field free region.

Figures 2 and 3 show the field and particle trajectory vs. position $z$ for the SXU magnet at a $31 \mathrm{~mm}$ gap. The particle trajectory $x(z)$ is the second integral of the field divided by the rigidity $B \rho$, defined according to $B \rho(\mathrm{kG}-\mathrm{m})=33.35 E(\mathrm{GeV})=84.3$ (kG-m) at $2.53 \mathrm{GeV}$. The variation of peak field with gap was observed to obey $B($ Tesla $)=0.534 / \sinh (\pi \times g a p / \lambda)$.

The data of figure 2 was taken after correcting the dipole field component using trim magnets at the magnet ends. Before compensation, the field integral was $=200 \mathrm{G}-\mathrm{cm}$, corresponding to $\approx 25 \mu \mathrm{rad}$ of angular deflection at $2.53 \mathrm{GeV}$, which is quite small. Note that the deviation of the trajectory from pure sinusoidal behavior is smaller than the 
oscillation amplitude. The field quality of the SXU magnet is more than satisfactory for the user's application.

\section{THE SUW MAGNET}

The superconducting wiggler magnet ${ }^{(3)(5)(6)}$ is composed of 5 poles powered in series (i.e. the main windings) having a peak field of $5.5 \mathrm{Tesla}$, and two end poles also powered in series (end windings) with a peak field of 3.1 Tesla. The wiggler poles and supporting structure are made of iron, and an iron field clamp is attached to each end of the wiggler to control fringe fields. The above fields are attained immediately prior to a magnet quench. The maximum field used during normal operation will be 5.22 Tesla (table 1). The main winding current will be ramped at $.9 \mathrm{~A} / \mathrm{s}$ from 1.15 to $4.72 \mathrm{Tesla}$, but must be slowed to $.2 \mathrm{~A} / \mathrm{s}$ between 4.72 and 5.22 Tesla to reduce the probability of a quench.

A continuously rotating long coil $(.29 \mathrm{~Hz})$ was used to measure the multipole coefficients for SUW. There were 3 sets of windings with different geometries to provide simultaneous independent measurements. The Fourier components of the time varying voltage measured across the coils yielded the integrated multipoles, according to equation (1). By varying the magnet end winding current, it was possible to reduce the integrated dipole coefficient to $\pm 700 \mathrm{G}-\mathrm{cm}$ at 6 different field levels using this method. Table 2 is a list of quadrupole and sextupole coefficients for the SUW for minimal dipole field integral. The measured quadrupole coefficient would be zero for a perfectly centered coil.

The main and end winding current settings for the SUW that result in minimal dipole field integral while ramping are different from the static settings. This is because the inductance of the main and end coils depends on current in a different way due to iron 
saturation, causing the end and main coil circuits to have different $L / R$ time constants (the magnet windings have parallel resistance inside the cryostat).

To determine the ramp current settings, a stationary long coil was placed normal to the field inside a metal bore tube along the magnet axis, and connecter to a voltage integrater. The integrator outpur was displayed on a chart recorder while ramping. Ramps having minimal integrated dipole field ( $\pm 200 \mathrm{G}-\mathrm{cm}$ ) were found. Fíp coil measurements at fixed current were also done using this arrangement.

Hall probe measurements were made using a probe mounted on the end of a steel tube. The tube had dowel pin holes positioned along its length at 0.25 inch intervals to facilitate systematic movements of the probe. A hose was run down to the probe through which heated nitrogen was delivered, for the purpose of temperature stabilization. Also, a temperature sensor was mounted near the probe. Figures 4 and 5 show field and displacement data for the SUW at 5.22 Tesla peak field with the dipole field integral compensated.

\section{THE HBW MAGNET}

The HBW magnets use a REC hybrid design with vanadium-permendur poles. The magnetization of individual HBW magnet blocks was determined with the Helmholtz coil - voltage integrator technique described earlier. Because there are eight magnet blocks in each slot, a higher degree of symmetry could be achieved in the final magnet configuration than for SXU. First, blocks were sorted by increasing strength of major component and arranged into pairs, strongest with weakest. The two magnets in one pair are located adjacent to one another in the $\mathbf{z}$ direction. The standard deviation of major component strength for pairs was $\pm 0.2 \%$, as opposed to $\pm 4 \%$ before pairing. 
The pairs were then sorted in order of increasing mean square strength of minor component. The pairs with the smallest minor component were placed closest to the magnet center line, the next smallest close to the back plate, and the pairs with largest minor component were buried inside, where they would be well shielded by nearby pole material. Symmetry was optimized, so that miror component magnetizations in the top and bottom beams were nearly mirror images, and their field integrals would cancel. The layers were organized so that the block minor components would appear as in figure 6 .

A total of $28 \mathrm{HBW}$ slots were loaded, with one slot left unloaded at each end to minimize fringe field effects. Figures 7 and 8 show preliminary field and displacement data for the HBW using a $62.5 \mathrm{~mm}$ gap. The peak field will be 1.1 Tesla with a $22 \mathrm{~mm}$ gap.

\section{ACKNOWLEDGEMENTS}

The authors are grateful to Klaus Halbach and Brian Kincaid for helpful discussions in the course of magnet construction and measurement.

\section{REFERENCES}

[1] H. Rarback, C. Jacobsen, J. Kirz, and I. McNulty, "The Performance of the NSLS Mini-Undulator", NIM, A266 , 96-105 (1988).

[2] W. Thomlinson, D. Chapman, N. Gmur, and N. Lazarz, "The Superconducting Wiggler Beamport at the National Synchrotron Light Source", NIM, A266, 226-233 (1988). 
[3] G. Decker, J. Galayda, "Magnetic Measurements on the X-17 Superconducting Wiggler", Informal Report BNL 40031 (1987).

[4] G. Brown, K. Halbach, J. Harris, H. Winick, "Wiggler and Undulator Magnets - a Review", NIM 208 , 65-77 (1983)

[5] A. Luccio, "Preliminary Measurements of Integrated Multipoles of the Superconducting Wiggler", NSLS Technical Note 233 (1985)

[6] A. Luccio, "Magnetic Measurements on the NSLS Superconducting Wiggler", NSLS Technical Note 186 (1984). 


\begin{tabular}{|c|c|c|c|}
\hline $\begin{array}{c}\text { INSERTION DEVICE } \\
\text { PARAMETERS }\end{array}$ & SXU & HBW & SUW \\
\hline Number of Poles & 77 & 31 & 7 \\
\hline Magnet Period $\lambda(\mathrm{cm})$ & 8.0 & 12.0 & 17.4 \\
\hline No. Magnet Blocks / Slot & 2 & 8 & - \\
\hline Peak Field $B_{\max }(\mathrm{kG})$ & 3.5 & 11.0 & 52.0 \\
\hline Max. Deflection Parameter K & 2.6 & 12.3 & 84.5 \\
\hline $\begin{array}{c}\text { Total Photon Power (kW) } \\
\text { @ 500 mA, 2.5 GeV }\end{array}$ & .72 & 4.3 & 24. \\
\hline
\end{tabular}

Table 1. X-ray ring insertion device parameters. 


\begin{tabular}{|c|c|c|c|c|}
\hline $\begin{array}{c}\text { B } \\
\text { (Tesla) }\end{array}$ & $\begin{array}{c}\operatorname{Re} c_{2} \\
\text { (Gauss) }\end{array}$ & $\begin{array}{c}\operatorname{Im} c_{2} \\
\text { (Gauss) }\end{array}$ & $\begin{array}{c}\operatorname{Re} c_{3} \\
(\mathrm{~T} / \mathrm{m})\end{array}$ & $\begin{array}{c}\operatorname{Im} c_{3} \\
(\mathrm{~T} / \mathrm{m})\end{array}$ \\
\hline \hline 5.22 & 11.6 & 62.8 & .509 & .093 \\
\hline 4.72 & 8.5 & 51.9 & .435 & .080 \\
\hline 3.94 & 5.1 & 45.5 & .367 & .061 \\
\hline 2.93 & 3.9 & 34.2 & .280 & .040 \\
\hline 1.88 & 3.5 & 7.2 & -.323 & .015 \\
\hline 1.15 & -1.1 & -2.2 & -.275 & .007 \\
\hline
\end{tabular}

Table 2. Multipole Coefficients for SUW. 


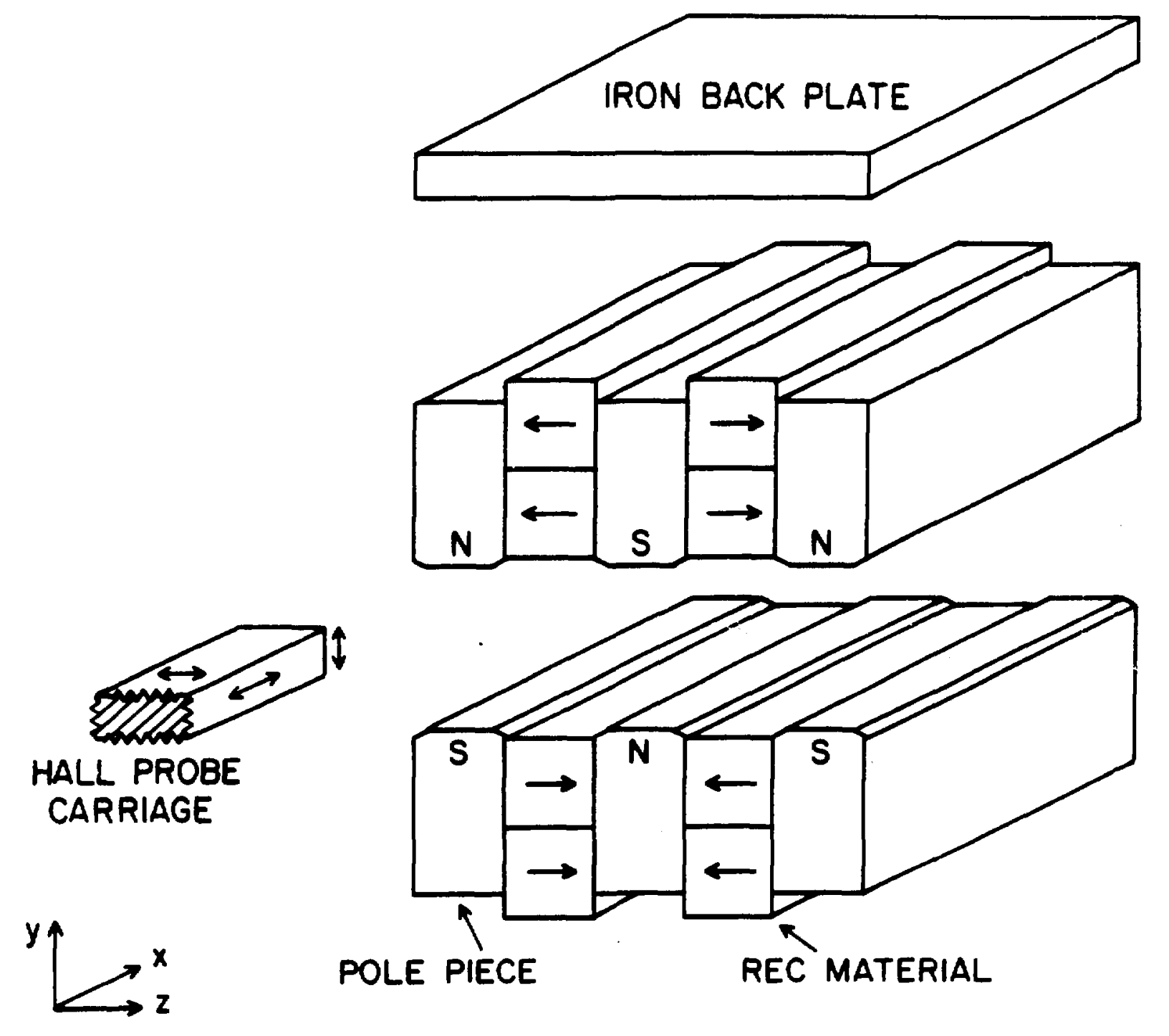

Figure 1. Arrangement for SXU Hall probe measurement. 


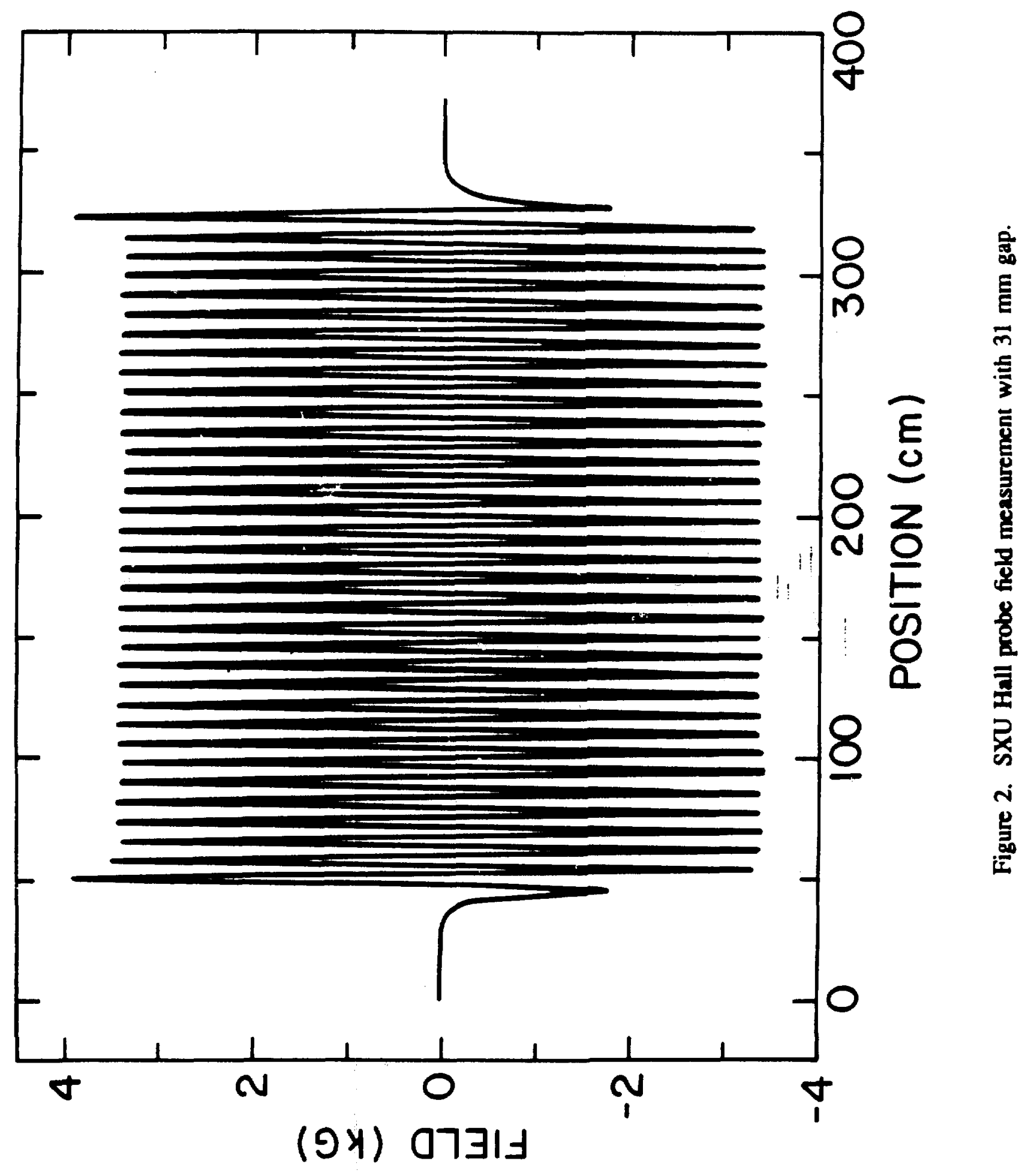




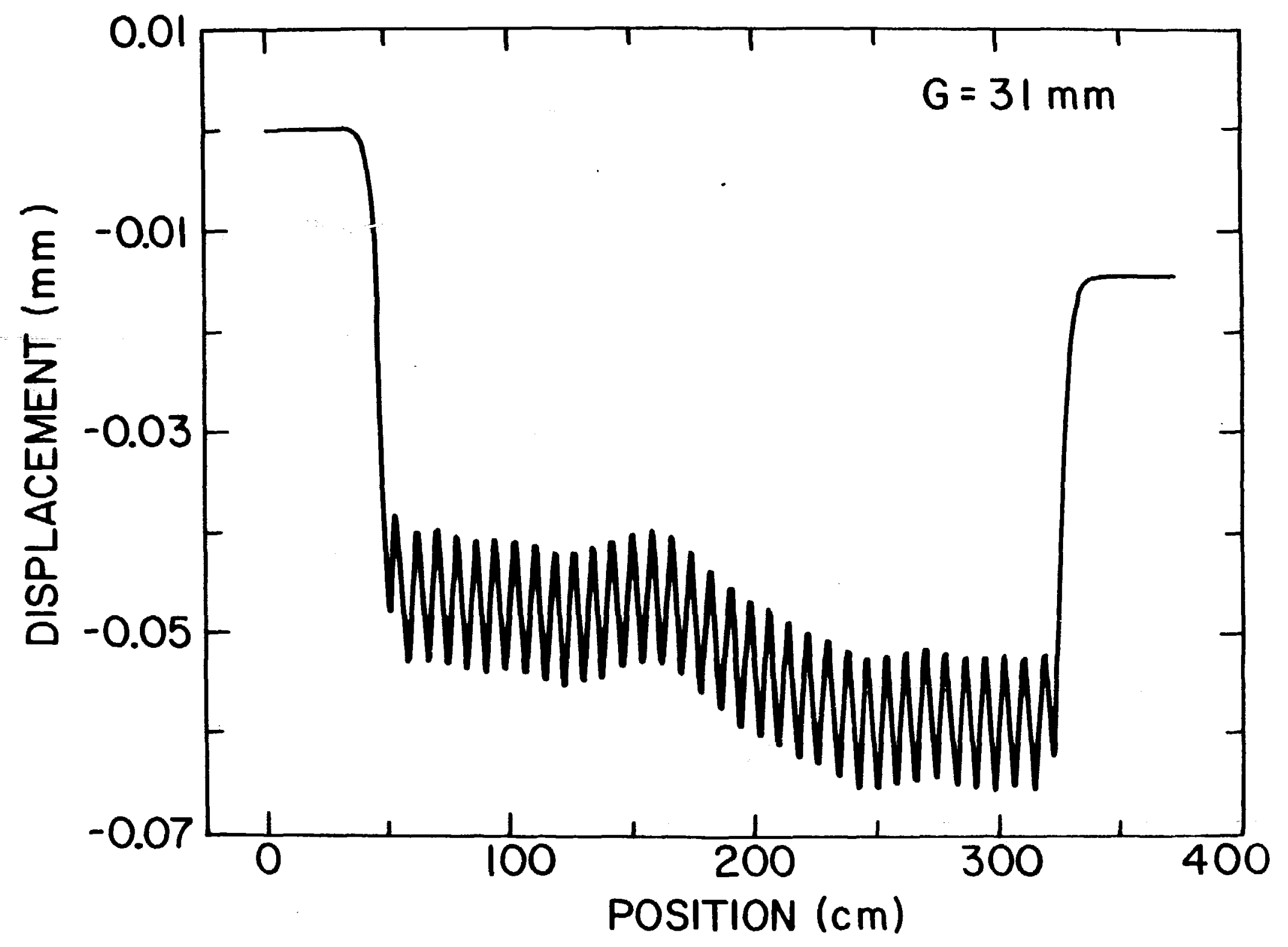

Figure 3. Particle trajectory in SXU caused by field of figure 2. 


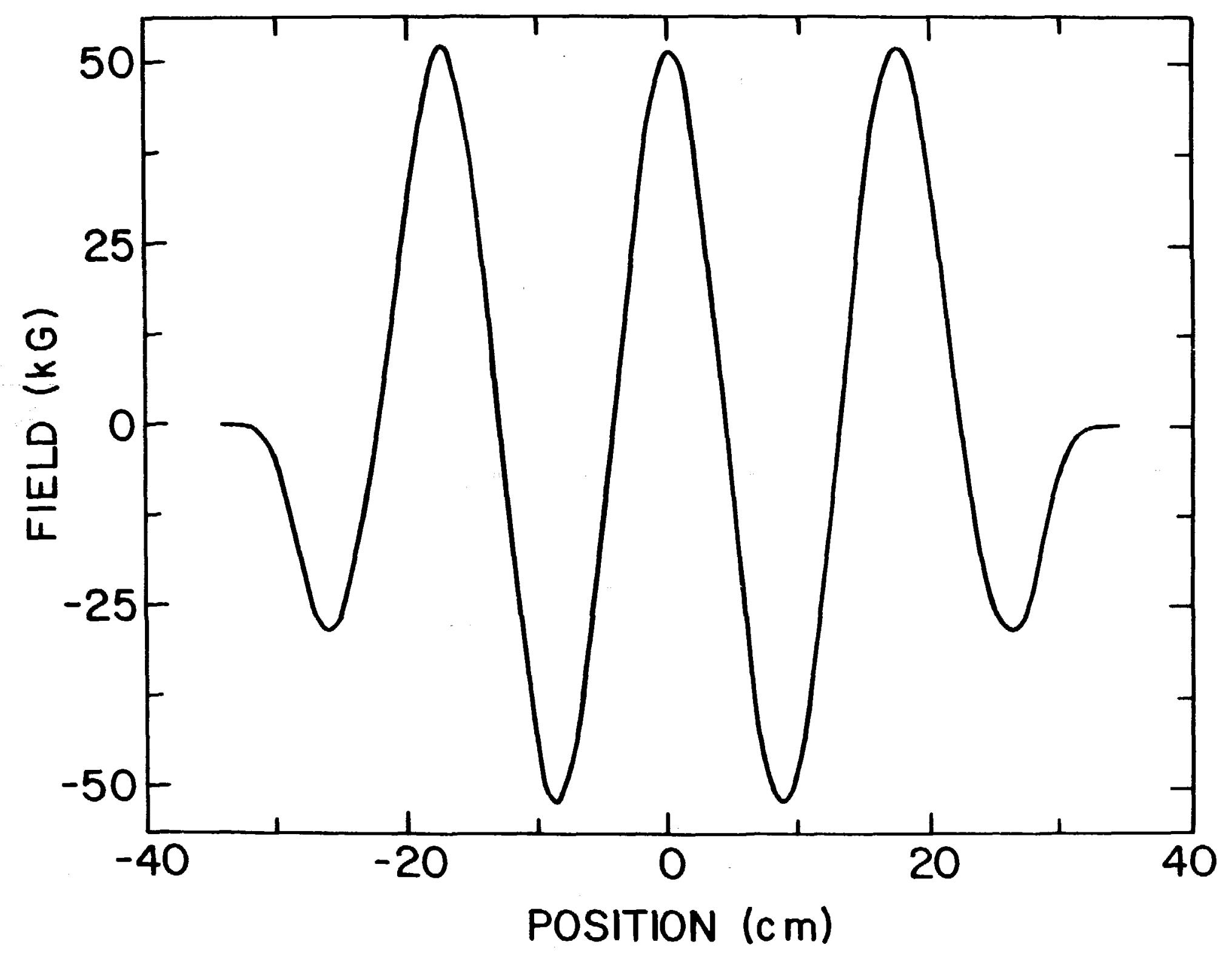

Figure 4. SUW Hall probe measurement at high field. 


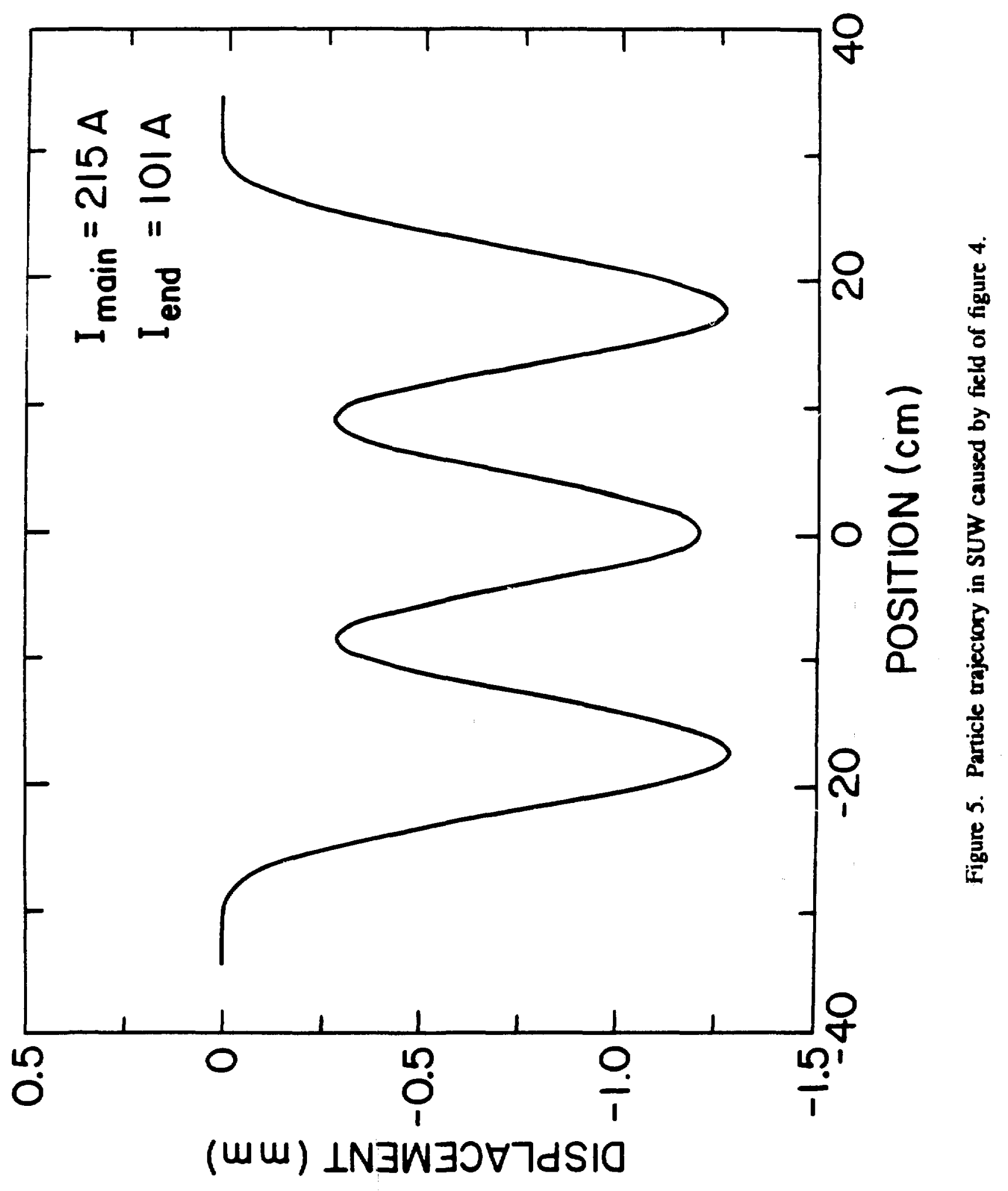



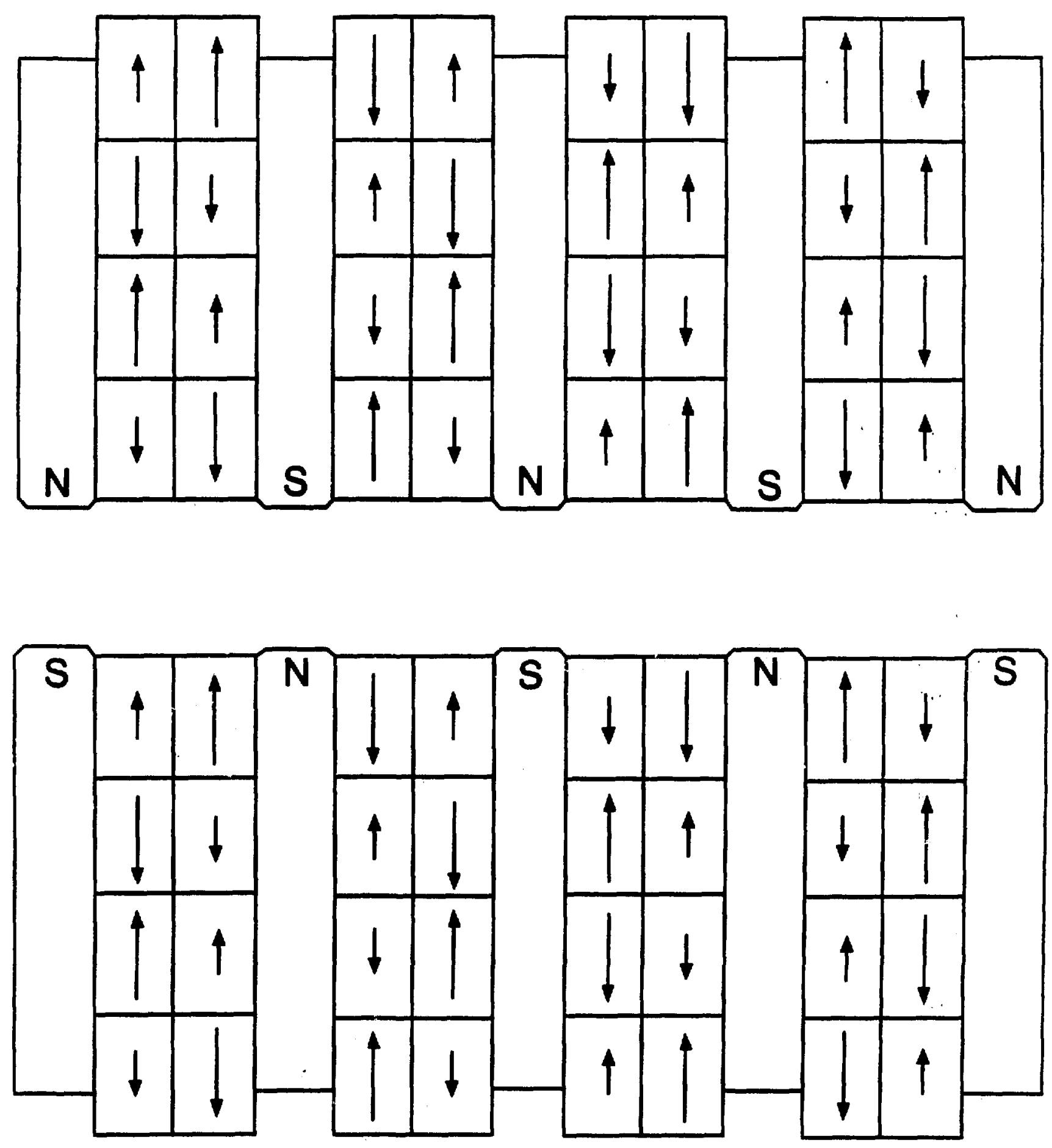

Figure 6. HBW loading pattern for minor component of magnetization. 


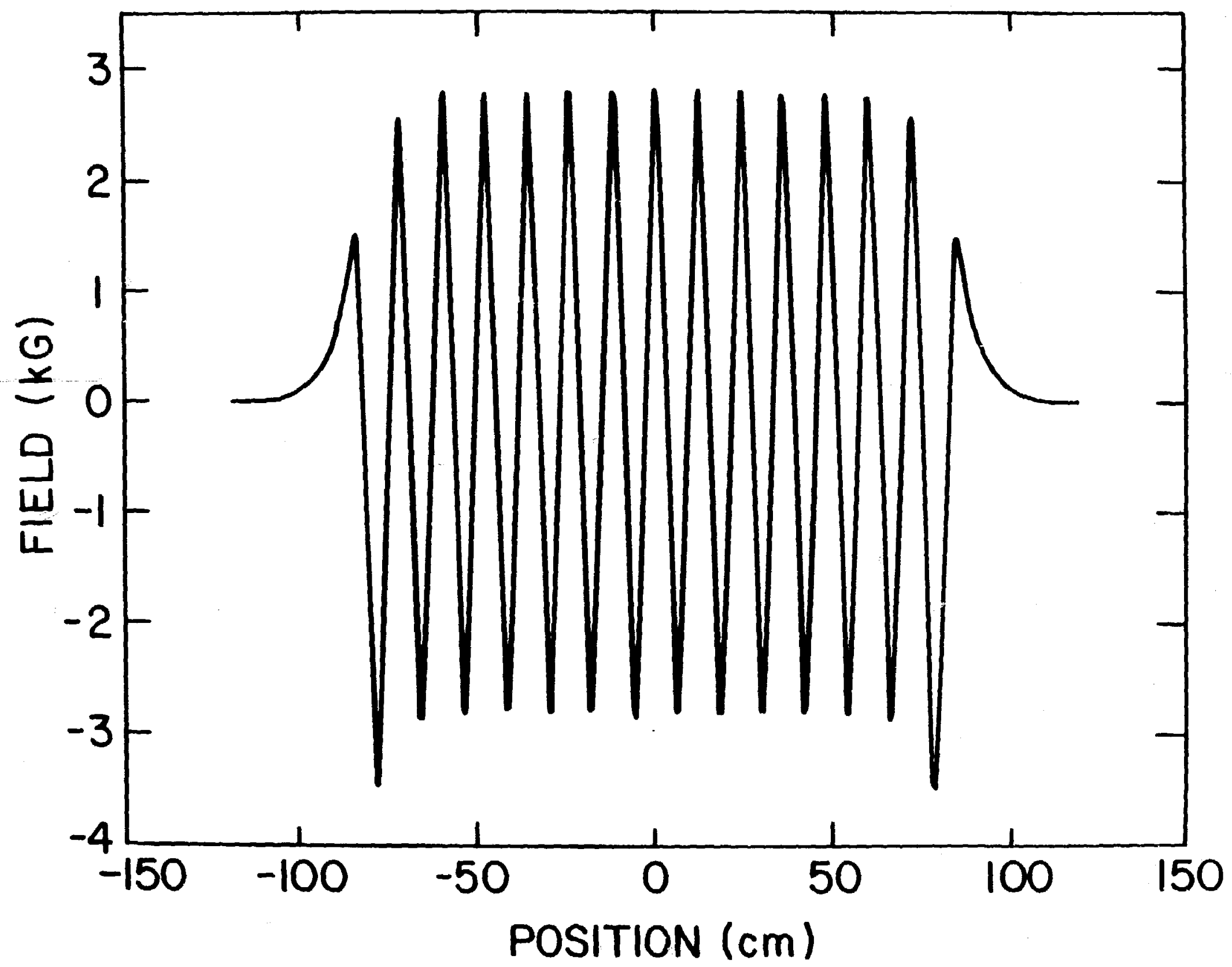

Figure 7. HBW Hall probe field measurement with $62.5 \mathrm{~mm}$ gap. 


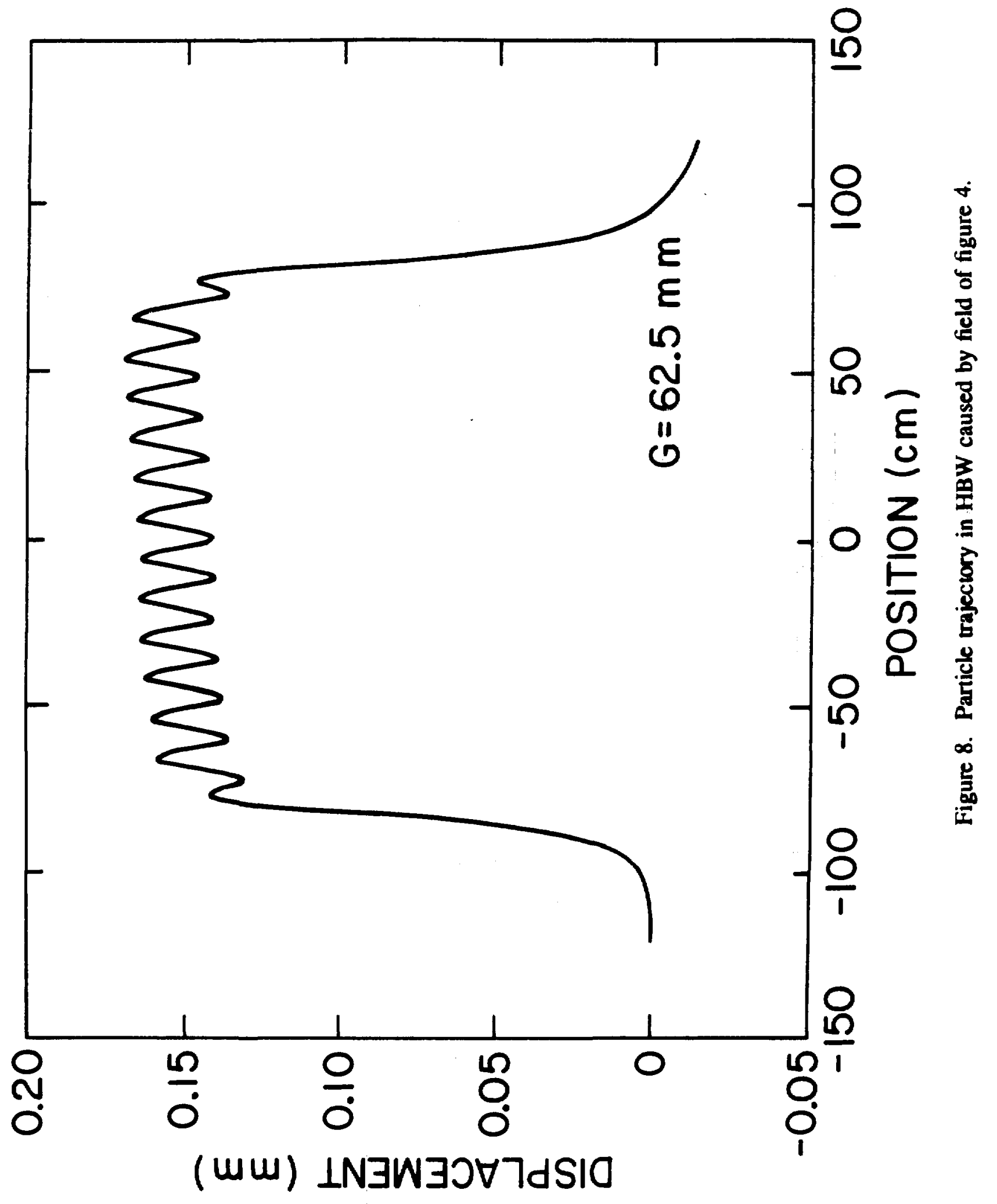

